\title{
FIRST RADIOACTIVE IONS CHARGE BRED IN REXEBIS AT THE REXISOLDE ACCELERATOR
}

\author{
$\underline{\text { B.H. Wolf }}^{\dagger}$ \\ Johannes Gutenberg-Universität Mainz, Germany \\ J. Cederkäll, O. Forstner, F. Wenander, \\ CERN, Switzerland \\ F. Ames, K. Reisinger \\ Ludwig-Maximilians-Universität München, Germany \\ L. Liljeby, Ö. Skeppstedt \\ Manne Siegbahn Laboratory, Sweden \\ B. Jonson, G. Nyman \\ Chalmers University of Technology Göteborg, Sweden,
}

And the REX-ISOLDE Collaboration

\begin{abstract}
REXEBIS is the charge breeder of the REX-ISOLDE post accelerator. Radioactive $1^{+}$ions produced at ISOLDE are accumulated, phase-space cooled and bunched in REXTRAP, and thereafter injected into the EBIS with an energy up to $60 \mathrm{keV}$.

The REXEBIS produced the first charge bred ions in August 2001 and has been running nearly non-stop from September to December 2001. It has delivered stable ${ }^{39} \mathrm{~K}^{10+}$ and ${ }^{23} \mathrm{Na}^{7+}$ beams generated in the testion-source in front of REXTRAP with a current of $12 \mathrm{pA}$ for $\mathrm{K}^{10+}$ and a $\mathrm{Na}^{7+}$ current exceeding $70 \mathrm{pA}$ $\left(6 \cdot 10{ }^{7} \mathrm{p} / \mathrm{s}\right.$ ). Stable ${ }^{27} \mathrm{Al}^{7+}$ and ${ }^{23} \mathrm{Na}^{6+}$ (about $10 \mathrm{pA}$ ) and also the first radioactive ${ }^{26} \mathrm{Na}^{7+}$ and ${ }^{24} \mathrm{Na}^{7+}$ beams (just $0.4 \mathrm{pA}$ or $5 \cdot 10^{5} \mathrm{p} / \mathrm{s}$ ), from the ISOLDE separator have been charge bred and accelerated for tests of the experimental setup. Despite some problems with the $\mathrm{LaB}_{6}$ cathode (one total breakdown after about 1500 hours of operation), displaying slow changes of the emission conditions, the EBIS is working remarkably stable.

We report about the first ion injections, ion source details, cathode conditions as well as other commissioning results.
\end{abstract}

Keywords: Radioactive ions, Charge breeding, EBIS, Ion trap.

PACS code: 29.25 . Ni

† EP Division-ISOLDE, CERN, CH-1211 Geneva, Switzerland. E-mail: Bernhard.Wolf@,cern.ch: Fax: +41 227678990 


\section{Introduction}

REXEBIS, the charge breeder of the REX-ISOLDE post accelerator, charge breeds $1^{+}$ ions that are phase-space cooled and bunched in REXTRAP.

The radioactive ions produced at ISOLDE are accumulated in the trap and thereafter injected into the EBIS with up to $60 \mathrm{keV}$ energy (the presently reached limit is $35 \mathrm{keV}$ ). The trap and the EBIS are normally operated with a repetition frequency of $50 \mathrm{~Hz}$. The ion injection into the EBIS consumes less than $50 \mu$ s and the breeding time is variable between 1 and $19 \mathrm{~ms}($ at $50 \mathrm{~Hz}$ ). In this way an optimal charge state with $\mathrm{A} / \mathrm{q}<4.5$ and small residual gas contamination can be selected. The extracted ion pulse length is between $10 \mu$ s and $50 \mu$ s depending on the extraction scheme. The highly charged ions are extracted from the EBIS at $5 \cdot \mathrm{A} / \mathrm{q} \mathrm{kV}$ (in order to match the injection criterion of the RFQ). Thereafter they are mass separated and finally accelerated in the REX-LINAC described in detail in another contribution to this conference [1]. Figure 1 shows a cross sectional view of the REXTRAP and EBIS set-up.

The complete REX-ISOLDE beam preparation system and LINAC was put into operation in fall 2001. The first physics experiments measuring Coulomb excitation and particle transfer reactions of neutron-rich radioactive sodium isotopes $\left({ }^{24} \mathrm{Na},{ }^{25} \mathrm{Na}\right)$ at 2.2 $\mathrm{MeV} / \mathrm{u}$ have already been performed. New experiments started in April 2002. Furthermore an energy up-grade to $3.1 \mathrm{MeV} / \mathrm{u}$ is underway.

\section{REXTRAP}

The experimental set-up of REXTRAP is described in detail in refs [2,3]. The cylindrical trap is located in the field of a $3 \mathrm{~T}$ superconducting solenoid on a high voltage platform having a voltage of up to $60 \mathrm{kV}$. Ne or Ar at a gas pressure of up to $10^{-3} \mathrm{mbar}$ is 
used as buffer gas in the trap centre. As the ions have to pass a small diaphragm at the trap exit, cooling, i.e. centring of the ions, can be detected by an increase in the extracted number of ions.

Experiments have been performed with stable and radioactive ions covering the mass range between ${ }^{7} \mathrm{Li}$ and ${ }^{238} \mathrm{U}$, using side-band cooling. An efficiency of about $40 \%$ has been reached for an accumulation and cooling time of totally 20 ms. Losses predominantly occur at the injection of the ions into the magnetic field of the trap. The transversal emittance of the ejected ion beam has been determined to $7 \pi \mathrm{mm} \operatorname{mrad}$ at $30 \mathrm{keV}$ compared to nearly $20 \pi \mathrm{mm}$ mrad without cooling. This concept works well if the number of stored ions per cycle is less than $10^{6}$. With more ions stored in the trap a significant decrease in the efficiency and an upward shift of the cooling frequency have been observed [4]. This indicates that the diameter of the ion cloud becomes larger than the extraction diaphragm and that side-band cooling is no longer sufficient for compressing the ion cloud in this regime. The shift of the frequency can be interpreted as a rotation of the ion cloud due to the higher space-charge density. For REX-ISOLDE, radioactive ion beam intensities will rarely reach this limit. The size of the ion cloud can however be further reduced by additional rotating wall compression [5].

\section{REXEBIS}

REXEBIS is an Electron Beam Ion Source consisting of a $500 \mathrm{~mA}$ electron beam that is compressed to $250 \mathrm{~A} / \mathrm{cm}^{2}$ in the $2 \mathrm{~T}$ field of a superconducting solenoid (for the results presented in this paper only $200 \mathrm{~mA}$ or $100 \mathrm{~mA} / \mathrm{cm}^{2}$ have been used). The whole length of the EBIS is about $2 \mathrm{~m}$ and the trapping region where the charge breeding occurs is 0.8 
$\mathrm{m}$. The maximal charge state for elements $\mathrm{A}<50$ results in an $\mathrm{A} / \mathrm{q}$ between 3 and 4 for a breeding time of $20 \mathrm{~ms}$. Elements heavier than $\mathrm{A}=50$ will need longer breeding times in order to reach sufficient intensities for $\mathrm{A} / \mathrm{q}<4.5$ required by the REX-LINAC. A detailed description of REXEBIS can be found in refs. $[6,7,8]$.

During the first half of 2001 an extensive up-grade of the REXEBIS took place as the previous tests showed some vacuum and structural problems [6]. Mapping and finetuning of the solenoid field reduced the electron beam losses (by more than a factor of 10) to less than $1 \%$. The perforation of the drift tubes with more than 2000 holes resulted in a significantly lower pressure inside the EBIS being now $5 \cdot 10^{-11}$ mbar with electron beam on and $5 \cdot 10^{-12}$ mbar without. The improved vacuum results in an increase in the compensation time of the electron beam (by restgas ions) to more than $1 \mathrm{~s} \mathrm{compared} \mathrm{to}$ only $50 \mathrm{~ms}$ in earlier measurements [8]. This allows for the longer breeding times necessary when breeding heavier elements $(\mathrm{A}>50)$ to $\mathrm{q} / \mathrm{A}<4.5$.

The inner structure of REXEBIS has also been modified to have 7 drift tubes, 3 of them for trapping. Four different combinations giving trapping lengths of 200, 400, 600 and $800 \mathrm{~mm}$ are now possible. Lanthanum and boron, evaporated from the cathode, ionised in the first drift tube can now be reflected back to the cathode by a short inner barrier. For $20 \mathrm{~ms}$ breeding time the intensity of $\mathrm{La}^{20+}$ ions is reduced from about 2 to $0.05 \mathrm{pA}$ with this design (see Figure 2).

REXEBIS delivered the first charge bred ions in August 2001 and ran nearly non-stop from September to December 2001. It has delivered stable ${ }^{39} \mathrm{~K}^{10+}$ and ${ }^{23} \mathrm{Na}^{7+}$ beams primarily produced in the test-ion-source in front of REXTRAP. An ion spectrum showing currents of ${ }^{23} \mathrm{Na}$ and $\mathrm{Ne}$ (the trap buffer gas) charge-bred in the REXEBIS is 
presented in Figure 3. The $\mathrm{Na}^{7+}$ current was $70 \mathrm{pA}\left(6 \cdot 10^{7} \mathrm{p} / \mathrm{s}\right)$. Stable ${ }^{27} \mathrm{Al}^{7+}$ and ${ }^{23} \mathrm{Na}^{6+}$ from ISOLDE and also the first radioactive ${ }^{26} \mathrm{Na}^{7+}$ and ${ }^{24} \mathrm{Na}^{7+}$ beams (about $5 \cdot 10{ }^{5} \mathrm{p} / \mathrm{s}$ ) have been charge bred and accelerated for detector tests (see Figure 4).

Despite of some problems with the $\mathrm{LaB}_{6}$ cathode the EBIS is working remarkably stable. Two cathodes had a mechanical failures after about 1500 hours of operation, due to magnetic forces when power was switched off abruptly. All cathodes display changes of the emission conditions and deteriorate slowly. An exchange of the cathode will probably be necessary every 2-3 month, but alternative cathode materials and new designs will be tested for more reliable and longer operational time. The theoretical lifetime of a $\mathrm{LaB}_{6}$ crystal itself would be more than one year determined by the evaporation rate at the temperature used.

\section{Conclusions}

REXEBIS has proven to be a suitable source for charge breeding of small amounts of radioactive ions to be economically accelerated by RF linacs. In connection with REXTRAP, an overall efficiency of $5 \%$ to $7 \%$ has been realised for the isotopes so far tested. More experiments using lithium and sodium beams will be continued during 2002.

\section{Acknowledgements}

The Knut and Alice Wallenberg Foundation, Sweden, funded the EBIS, the European Union supported the REX-ISOLDE collaboration under contract no. HPRI-CT-19950003. The authors would also like to thank the ISOLDE and PS technical team for their support during construction and commissioning of REX. 


\section{$\underline{\text { References }}$}

[1] O. Kester et al., this Proceedings

[2] F. Ames et al., Proc. Exotic Nuclei and Atomic Masses, Bellaire, USA, 1998,

AIP Conf. Proc. 455927 (1998)

[3] P. Schmidt et al., Nucl. Phys. A 701, 550c (2002)

[4]. F. Ames et al., Hyperfine Interactions 132469 (2001)

[5]. F. Anderegg et al., Phys. Rev. Lett. 814875 (1998)

[6] F. Wenander et al,. "REXEBIS, design and initial commissioning results", Proc. of the $8^{\text {th }}$ International Symposium on EBIS and EBIT and their Applications, Upton, NY, USA, Nov. 2000, AIP-Conference-Proceedings, no.572, (2001) p.59-73.

[7] F. J. C. Wenander, "Charge Breeding and Production of Multiply Charged Ions in EBIS and ECRIS", Doctoral thesis, Chalmers University of Technology, Gothenburg, Sweden (2001), ISBN 91-7291-009-7, and CERN-THESIS-2001-005.

[8] B. H. Wolf, J. Cederkäll, F. Wenander et al., "Commissioning results from the REXEBIS charge breeder", Proc. $9^{\text {th }}$ Int. Conf. On Ion Sources, Oakland, California USA 2001, Rev. Sci. Instrum. 73, 682 (2002) 


\section{Figures}

FIGURE 1: The layout of accumulator, buncher and charge breeder.

FIGURE 2: Suppression of ions coming from the cathode area by increasing the inner EBIS barrier potential by $200 \mathrm{~V}$. Tube down means no barrier between cathode and breeding area, tube up potential barrier activated (filled area).

FIGURE 3: Ion spectrum for ${ }^{23} \mathrm{Na}$ from REXTRAP. The solid part is the EBIS background (potassium contamination from the trap).

FIGURE 4: Ion spectrum for ${ }^{26} \mathrm{Na}$ from ISOLDE. The current of $\mathrm{Na}^{7+}$ was $0.6 \mathrm{pA}$ corresponding to $5 \cdot 10^{5}$ particles per second. 


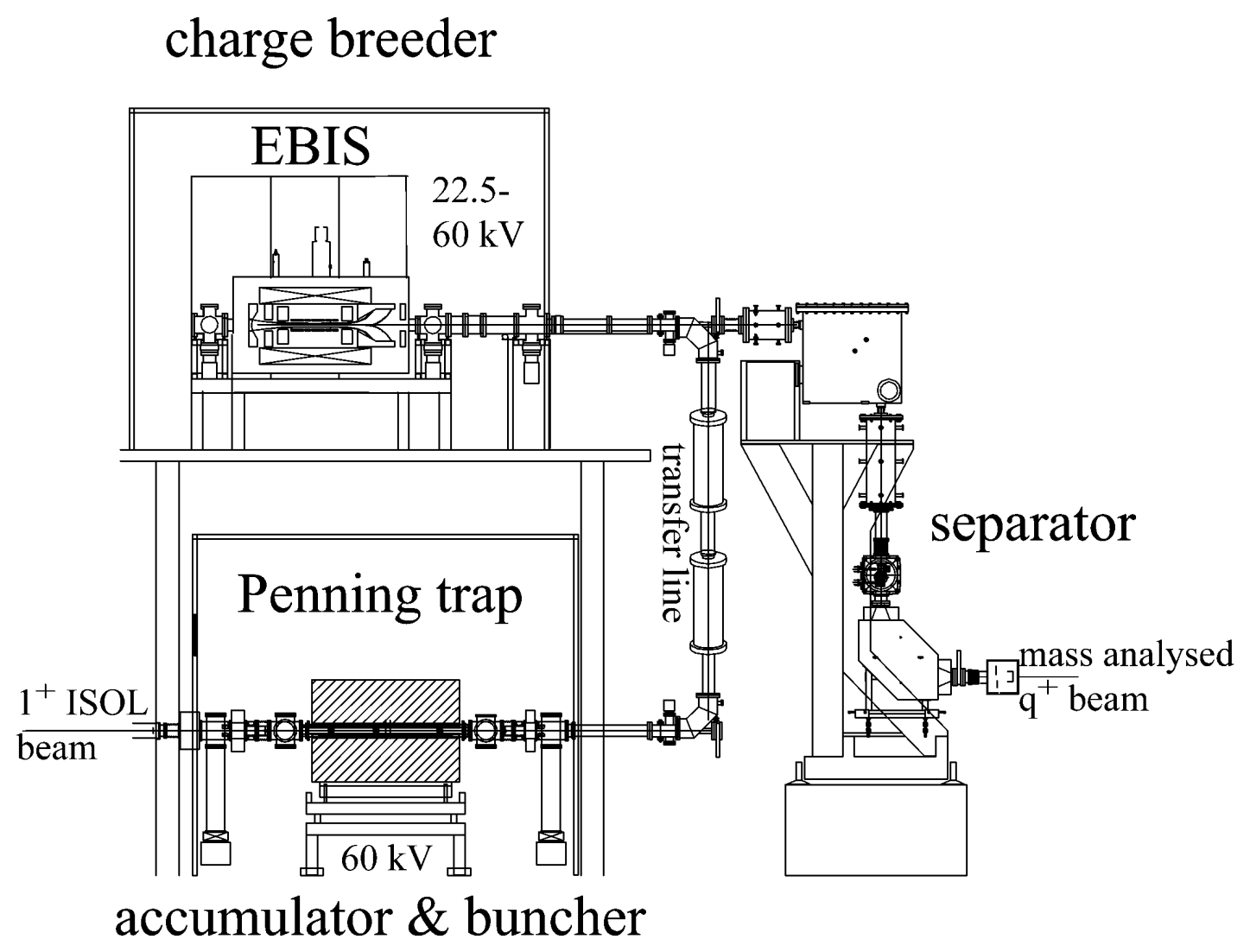

FIGURE 1. 


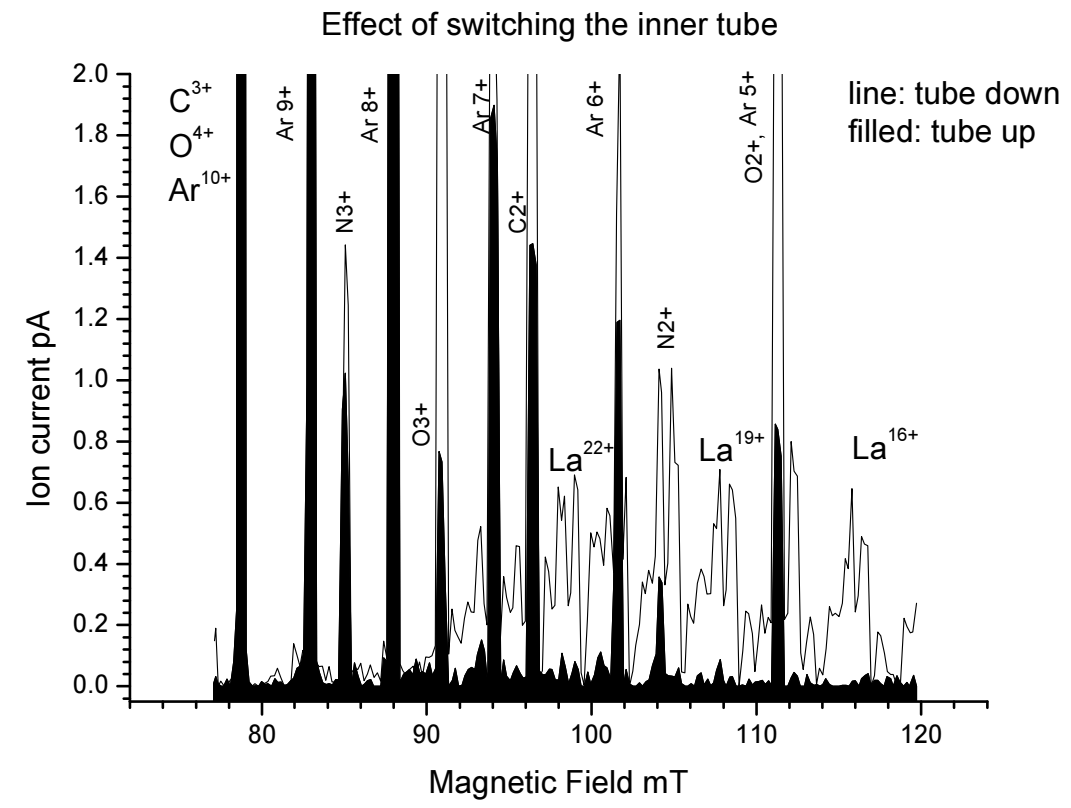

FIGURE 2. 
20/11/01

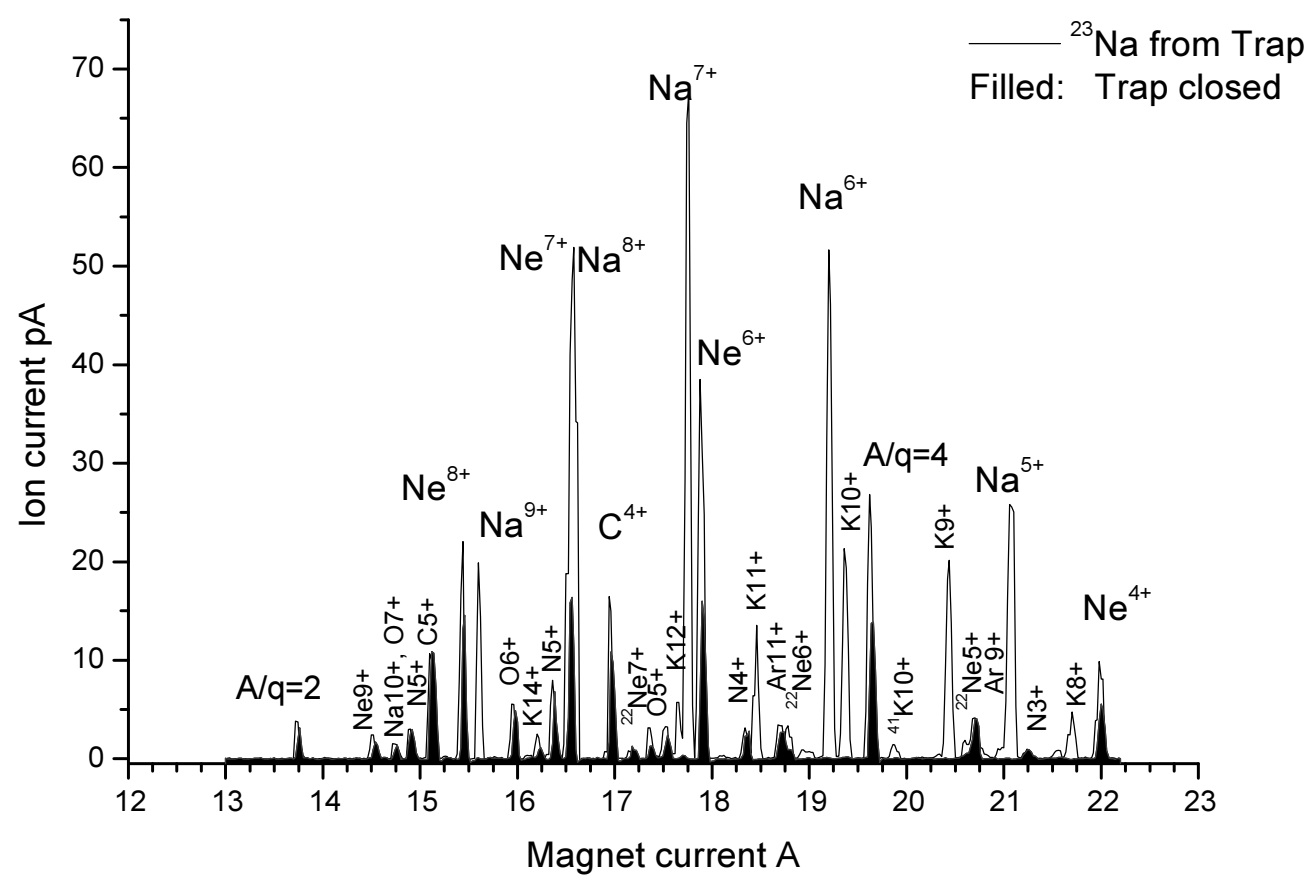

FIGURE 3. 
02/11/01

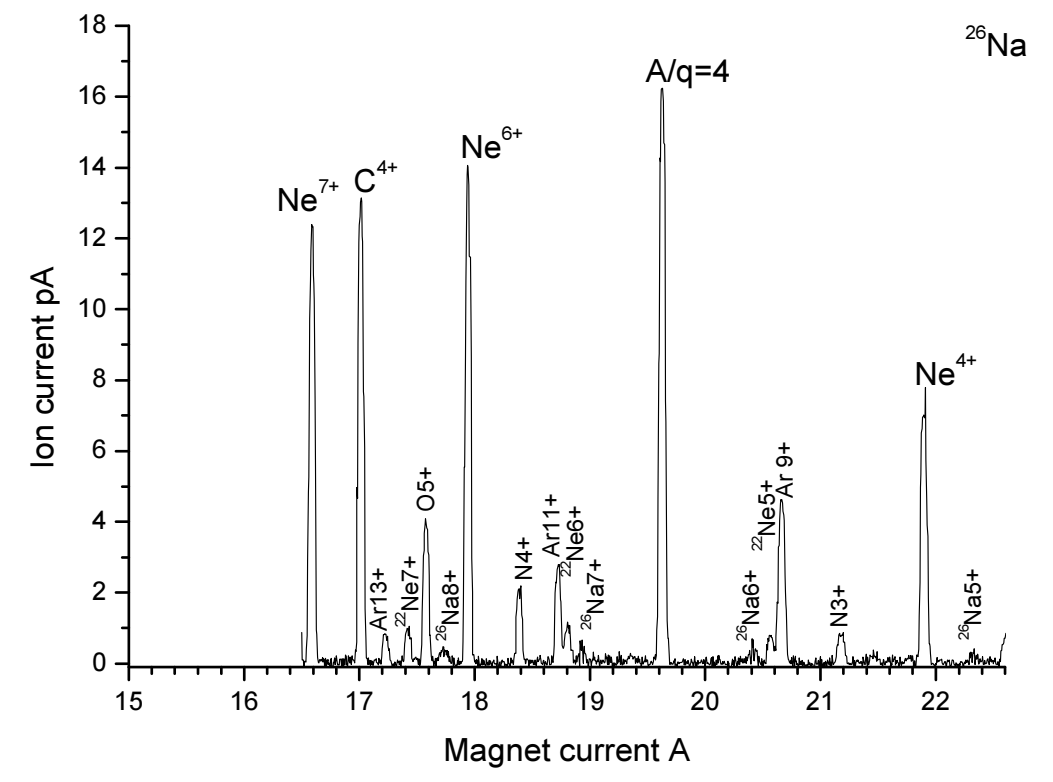

FIGURE 4. 